\title{
VULNERABILIDADE DA VEGETAÇÃO DE CAMPOS DE ALTITUDE ÀS MUDANÇAS CLIMÁTICAS
}

\author{
Mayara Vescovi Assis ${ }^{1 *}$ \& Eduardo Arcoverde de Mattos ${ }^{1}$ \\ ${ }^{1}$ Universidade Federal do Rio de Janeiro (UFRJ), Instituto de Biologia, Departamento de Ecologia, Programa de Pós-Graduação em \\ Ecologia, Laboratório de Ecologia Vegetal, Av. Brigadeiro Trompowski, s/n, Cidade Universitária, Ilha do Fundão, Rio de Janeiro, \\ RJ, Brasil. CEP: 21941-599. \\ E-mail: mayara.assiis@gmail.com; eamattos@gmail.com.
}

\section{RESUMO}

Estudos têm demonstrado que ambientes de montanhas experimentam um efeito maior das mudanças climáticas do que as porções mais baixas do continente. Neste contexto, as montanhas tropicais têm se destacado como potencialmente as mais suscetíveis, com efeito direto sobre a biota associada. Entretanto, nenhum estudo que buscasse estimar a vulnerabilidade dos campos de altitude inseridos na Mata Atlântica frente às mudanças climáticas foi realizado até hoje. Com este objetivo em mente, realizamos uma síntese da literatura e das previsões climáticas para 2050 a fim de responder a três questões: 1) quais são as previsões de mudanças no clima para os campos de altitude da região sudeste do Brasil? 2) com base no que já foi observado e estimado para ambientes similares, como a vegetação de campos de altitude será afetada em um cenário de mudanças climáticas? 3) quais fatores tornam vulnerável a vegetação de campos de altitude a estas alterações? Segundo o GCM HadGEM2-ES, esperam-se aumentos significativos nas temperaturas médias anuais e aumentos pouco expressivos na precipitação anual nos campos de altitude da região sudeste do Brasil. Alterações na sazonalidade climática também serão sutis. Apesar de não terem sido encontrados estudos que testem diretamente os prováveis efeitos das mudanças climáticas na vegetação de campos de altitude, vários trabalhos apontam a vulnerabilidade desta vegetação a alterações no clima. Observou-se que a vulnerabilidade da vegetação de campos de altitude está relacionada à distribuição restrita e disjunta da vegetação, bem como à sua ocorrência em uma restrita faixa altitudinal. Além desses fatores, foram apontados na literatura a suscetibilidade da vegetação à ocorrência de incêndios e os altos índices de endemismo observados nestas formações. Entretanto, os estudos ecológicos abordando essa temática ainda são escassos, o que compromete a capacidade de previsão de resposta da vegetação às futuras mudanças no clima. Sugerimos uma complementaridade entre estudos manipulativos e naturais para avaliar os efeitos de alterações antrópicas para as respostas da vegetação às mudanças climáticas, buscando diferenciar os fatores que afetam a distribuição e abundância das espécies endêmicas e a contribuição relativa dos fatores de risco para um provável declínio dos campos de altitude.

Palavras-chave: aquecimento global; conservação; montanhas; vegetação de altitude; vulnerabilidade endógena.

\section{ABSTRACT - VULNERABILITY OF BRAZILIAN HIGH-ALTITUDE GRASSLANDS TO CLIMATE CHANGE}

Many studies have established that mountains environments have experienced a larger effect of climate change than lower altitude areas. Despite the few studies realized, the tropical mountains are more vulnerable, which directly affect its biodiversity. However, no study aiming to investigate the vulnerability of campos de altitude in a climate change scenario was realized until today. With this goal in mind, we conduct a review in order to respond three questions: 1) what are the climate change forecasts for southeast campos de altitude? 2) Based in another studies about climate change and mountains, how the campos de altitude vegetation will be affected in a climate change scenario? 3) Which factors make the campos de altitude vegetation vulnerable in that scenario? According to GCM HadGEM2-ES, a significant increase in annual average temperature and less expressive increase in annual precipitation are expected for campos de altitude sites in southeast Brazil. Changes in climatic seasonality will be also subtle. No papers about the effects of climate change in campos de altitude vegetation were found. However, many studies have pointed out a probable vulnerability of this kind of vegetation to climate change. Vulnerability of campos de altitude vegetation is related to its restricted and disjoint geographical distribution, their restricted altitudinal range, the vegetation susceptibility to fires and high level of endemism. However, ecological studies about this theme are still scarce. Hence, lack of knowledge hinder predictability about the future response of 
campos de altitude vegetation to climate change. We suggest a complementary approach between manipulative and natural experiments in order to evaluate the effects of anthropic activities for vegetation responses and functional ecology of species. Additionally, we must detangle the factors that affect the distribution and abundance of endemic species and the relative contribution of the risk factors for vegetation probably decline under future climate change scenario.

Keywords: conservation; endogenous vulnerability; global warming; Highland Vegetation; mountains.

\section{INTRODUÇÃO}

De acordo com o Painel Intergovernamental de Mudanças Climáticas (Intergovernmental Panel on Climate Changes, IPCC) (2013), o clima do planeta tem sido afetado dramaticamente nas últimas décadas como produto da atividade humana. Dentre as variáveis climáticas mais estudadas e afetadas destacam-se a temperatura e a precipitação anual, com projeções de aumentos na média global nos próximos anos (IPCC 2013). Estima-se que estas mudanças tenham um impacto negativo sobre a biodiversidade ao redor do globo, com consequências diretas sobre a ecologia e evolução das espécies (Bellard et al. 2012). Impactos sobre a fenologia, distribuição, e abundância das espécies são esperados e amplamente difundidos na literatura científica para diferentes biomas e regiões do globo (Marengo 2006, Hanna 2011), o que em alguns casos pode levar a extinção de populações das espécies mais suscetíveis a essas mudanças, alterando a estrutura das comunidades e o funcionamento dos ecossistemas. É importante destacar que a vulnerabilidade de ecossistemas e biomas às mudanças climáticas futuras pode ser amplificada pelo efeito de outras alterações antrópicas como o desmatamento (Malhi et al. 2008). Entretanto, pouco ainda se sabe sobre as consequências das mudanças climáticas para os biomas e ecossistemas brasileiros, assim como para ambientes tropicais em geral (Vale et al. 2009, Kreyling et al. 2010, PMBC 2014).

É consenso dentro da comunidade científica que os ambientes de montanha ao redor do globo são altamente sensíveis às mudanças climáticas. Autores como Beniston \& Stofell (2013) defendem que esta alta sensibilidade confere a estes ambientes o título de "sentinelas das mudanças climáticas". Isso porque existem evidências que indicam que as montanhas experimentam mudanças mais rápidas e marcantes no clima quando comparadas a porções mais baixas do continente (Beniston et al. 1997, Rangwala \& Miller 2012). Além disso, características como os altos índices de endemismo e o seu papel para manutenção e regulação do ciclo hidrológico dão a estes ambientes grande importância social e ambiental, além de os tornarem prioritários para a conservação (Beniston 2006, Kohler et al. 2010). Contudo, apesar da vulnerabilidade e importância destes ambientes, poucos estudos com esta temática têm sido realizados em montanhas tropicais.

Isolados nos picos das montanhas do sudeste brasileiro e associados ao bioma Mata Atlântica ocorrem os Campos de altitude que são campos úmidos subalpinos (Safford 1999a). A vegetação dos Campos de altitude é constituída predominantemente por gramíneas e arbustos, com elevado número de espécies endêmicas (Martinelli 1996, Safford 1999a). Segundo Safford (1999b, 2007), essas formações assemelham-se em relação ao clima e a florística aos Páramos Andinos. Entretanto, os campos de altitude possuem características climáticas que conferem singularidade às suas formações vegetais, como a sazonalidade climática fortemente marcada, ocorrência de temperaturas mais baixas e o comprimento do dia, que é maior no verão e menor no inverno (Safford 1999b). São apontados como ambientes sensíveis à atividade antrópica, especialmente a remoção da cobertura vegetal e também a mudanças no clima (Martinelli 2007). Por conta disso, uma das ações prioritárias do Programa Nacional de Pesquisas e Conservação em Ecossistemas de Montanhas (CONABIO 2008) é promover estudos de longa duração para monitoramento das mudanças climáticas e efeitos das ações antrópica em ecossistemas de montanhas. Contudo, apesar de sua importância biológica e de seu papel na manutenção de serviços ecossistêmicos, como por exemplo, o suprimento de água para população, poucos 
estudos têm sido desenvolvidos nestes ambientes (Safford 1999).

Desta forma, este trabalho teve como objetivo sintetizar o conhecimento existente sobre o grau e os principais fatores de vulnerabilidade da vegetação de campos de altitude às mudanças climáticas previstas para as próximas décadas. Para tanto, buscamos aqui responder a três questões principais: 1) quais são as previsões de mudanças no clima para os campos de altitude da região sudeste do Brasil? 2) com base no que já foi observado e estimado para ambientes similares, como a vegetação de campos de altitude será afetada em um cenário de mudanças climáticas? 3) quais fatores tornam vulnerável a vegetação de campos de altitude a estas alterações?

\section{MATERIAL E MÉTODOS}

A fim de ser responder as questões de interesse, foi realizada uma revisão bibliográfica focada na busca de artigos dentro da temática do trabalho. A principal plataforma de busca utilizada para acessar a literatura de interesse foi o SCOPUS com eventual acesso aos indexadores WEB OF SCIENCE e SCIELO para complementação da pesquisa. Além do uso de bases de busca, alguns artigos foram acessados através do uso de referências cruzadas contidas na literatura obtida através das plataformas de busca. Também foram utilizados artigos de conhecimento prévio dos autores deste estudo que fossem úteis e ou complementares a temática principal. Além da revisão bibliográfica, foi realizada também a síntese das previsões de alterações climáticas para o futuro em campos de altitude.

A primeira etapa da revisão constou numa busca preliminar da literatura existente para campos de altitude, com foco na vegetação e/ou trabalhos que abordassem questões ecológicas e em especial, a temática mudanças climáticas. As palavras chaves utilizadas nesta primeira etapa foram: campos de altitude, brazilian paramos associadas às palavras vegetation e plant. Nesta primeira busca já se pôde notar a reduzida literatura existente para os campos de altitude. Grande parte dos trabalhos encontrados foi descritiva, muitas vezes eram a respeito de um novo registro de espécie ou inventário de espécies. Nenhum artigo sobre os efeitos das mudanças climáticas sobre os campos de altitude foi encontrado. Devido à inexistência de trabalhos dentro da temática mudanças climáticas, a segunda parte da busca focou na procura de trabalhos que abordassem esse tema em regiões e vegetações análogas do globo. Procurou-se especificamente registros dos possíveis impactos das alterações previstas no clima sobre ambientes alpinos. As palavras chaves utilizadas nesta busca foram: Climate/Climatic change associadas às palavras mountains, vegetation, tropical mountains e highland vegetation. Devido ao grande número de artigos gerados a partir desta busca, foram priorizados aqueles com maior número de citações e/ou contidos em revistas de alto fator de impacto. No total foram lidos 61 artigos e livros para o desenvolvimento desta revisão, sendo apenas 18 específicos de vegetação de campos de altitude.

A terceira e última etapa do trabalho focou na síntese das previsões de seis variáveis bioclimáticas para o futuro (2050). As variáveis foram obtidas através do WorldClim (http://www. worldclim.org/) em dois recortes temporais, um no presente e outro para 2050, ambas na resolução de 30" (aproximadamente $1 \mathrm{~km}^{2}$ ). As variáveis bioclimáticas utilizadas foram a temperatura média anual, precipitação anual, temperatura média do trimestre mais quente, temperatura média do trimestre mais frio, precipitação do trimestre mais chuvoso e precipitação do trimestre mais seco (códigos bio1, bio12, bio10, bio11, bio16 e bio17, respectivamente). Devido à ocorrência de sazonalidade marcada nos campos, escolhemos também variáveis que refletissem a sazonalidade anual. Como o caso das médias de temperatura dos trimestres mais quente e frio e de precipitação dos trimestres mais seco e úmido. Foram assumidos dois cenários extremos de concentração de gases de efeito estufa segundo o IPCC (2013), sendo um cenário otimista (RCP2.6) e outro pessimista (RCP8.5). Os Representative Concentration Pathways (RCPs) são possíveis cenários futuros baseados, por exemplo, na emissão antrópica de gases do efeito estufa e no uso da terra (van Vuuren et al. 2011). As seis variáveis bioclimáticas futuras foram geradas a partir do modelo de circulação geral do Met Office Hadley Centre, HadGEM2ES (HadGEM2 Development Team 2011). As mudanças das seis variáveis foram medidas 
através da média das diferenças dos valores de cada variável nos dois recortes temporais. A fim de restringir as análises para as formações de campos de altitude, foram consideradas apenas as áreas localizadas a $1800 \mathrm{~m}$ de altitude ou mais na região sudeste. As análises foram realizadas através do pacote raster (Hijmans 2015) do software R (http:// cran.r-project.org/).

\section{MUDANÇAS NO CLIMA EM AMBIENTES DE MONTANHA}

Grande parte dos artigos utilizados nesta revisão corresponde a estudos desenvolvidos em regiões temperadas, especialmente em montanhas europeias, onde há um corpo de conhecimento mais sólido acerca do assunto. O número de trabalhos com enfoque em montanhas tropicais foi pouco expressivo. Entretanto, fomos capazes de incluir estudos desenvolvidos em grande parte do globo, com destaque para trabalhos realizados no continente Africano e na América do Sul (especialmente sobre os Andes), e em menor número trabalhos na Ásia (China) e Havaí. Portanto, alcançamos uma adequada representatividade geográfica e mais do que uma exaustiva busca pela totalidade de artigos publicados sobre o tema, buscamos estabelecer o que tem sido considerado como o entendimento geral em relação aos impactos das mudanças climáticas em ambientes de montanha. Desta forma, apesar da dificuldade de se realizar previsões para ambientes de montanha e da grande variabilidade de características dos mesmos entre diferentes regiões (Beniston 1997, 2006), existem evidências de que estes ambientes estão associados a algumas particularidades em relação às mudanças no clima. Alguns estudos demonstram que as montanhas estão experimentando um aquecimento maior e mais rápido do que porções mais baixas do continente e em relação à média global (Beniston et al. 1997, Beniston 2006). Embora ainda poucos, existem indícios de uma relação direta entre o aumento da temperatura e o aumento da elevação, que parece ser mais forte em altitudes mais elevadas (a partir de 3000m) (Rangwala \& Miller 2012). Aumentos nas temperaturas máximas e especialmente nas mínimas anuais e diárias são esperados e já têm sido observados para ambientes de montanha
(Sanz-Elorza et al. 2003, Beniston et al. 1997). Da mesma forma que já se observa um aumento maior das temperaturas no inverno do que no verão (Sanz-Elorza et al. 2003). Espera-se ainda que com o aumento da temperatura, o degelo naquelas montanhas que possuem cobertura de neve ocorra de forma acelerada, acarretando alterações significativas no ciclo hidrológico (Beniston \& Stofell 2013). Contudo, as particularidades topográficas, geológicas e microclimáticas dos ambientes de montanha muitas vezes não são levadas em consideração para realização destas previsões, o que dificulta a busca de generalizações (Beniston et al. 1997).

No caso das montanhas tropicais, esperase que as alterações climáticas, bem como os impactos dessas mudanças sob a biodiversidade, sejam maiores (Beniston et al. 1997). Essa vulnerabilidade está associada ao fato de montanhas tropicais serem mais sensíveis a alterações nas temperaturas da superfície dos oceanos ( Beniston et al. 1997, Pounds et al. 1998). Provavelmente por isso, a intensificação de eventos climáticos extremos como o El niño e La niña também tem sido apontada como uma das consequências previstas das mudanças climáticas (Beniston et al. 1997, Pounds et al. 1998). Nos Andes, uma taxa de aumento da temperatura de $0,34^{\circ} \mathrm{C}$ por década foi registrada durante o período de 1974 a 1998, bem como a ocorrência de anomalias dos fenômenos El niño e La niña (Vuille \& Bradley 2000). É bem provável que este aumento observado nas médias anuais de temperatura esteja relacionado à retração de glaciais já registrados para a região (Beniston et al. 1997, Vuille \& Bradley 2000).

Segundo Kohler et al. (2014), as alterações climáticas já observadas e as esperadas nas próximas décadas em ambientes de montanha são de preocupação e responsabilidade global. De acordo com o mesmo autor, isto se deve ao fato de que as consequências de tais alterações se estendem para além das próprias montanhas, apresentando um impacto indireto sobre outras regiões do planeta. A redução na disponibilidade de água em porções baixas do continente, por exemplo, é apontada por Kohler et al. (2014) como um dos principais impactos das mudanças climáticas sobre ambientes de montanha, através de alterações no ciclo hidrológico. De forma similar, o impacto 
das mudanças climáticas sobre a biodiversidade associada às montanhas merece atenção global, uma vez que nestes ambientes concentram-se grande quantidade dos hotspots de biodiversidade do mundo (Kohler et al. 2014).

\section{RESPOSTAS BIOLÓGICAS E FATORES DE RISCO DA VEGETAÇÃO DE ALTITUDE}

As elevações das temperaturas mínimas diárias e das temperaturas do inverno podem ser preocupantes e causar impactos diretos sobre a ecologia das espécies associadas às montanhas do globo. Mudanças nos regimes de precipitação e intensificação de eventos climáticos extremos também poderão apresentar impactos sobre estas espécies. Através de modelagem de nicho ecológico de plantas da Europa para o futuro, os trabalhos de Thuiller et al. (2004) e Engler et al. (2011) observaram que espécies alpinas são potencialmente mais sensíveis às mudanças climáticas do que as espécies de porções baixas do continente. Segundo os autores, isto se reflete em previsões de taxas de extinções desproporcionais (perda de espécies estimadas entre 5 a 60\%) em regiões de montanha da Europa até o fim do século. Dentre as respostas esperadas e já relatadas para espécies vegetais, destacam-se alterações na distribuição geográfica (Engler et al. 2009), mudanças na composição e alterações nos padrões de dominância da comunidade (Harte \& Shaw 1995, Baron et al. 2000, Angelo \& Daehler 2013), alterações ecofisiológicas (Shaw et al. 2000) e fenológicas (Harte et al. 1995). Além de previsões de extinções de populações de espécies de topo de montanha (Colwell et al. 2008). A maioria dos estudos concentra-se nas particularidades climáticas e topográficas das montanhas e pouco se sabe sobre os fatores biológicos associados a esta suscetibilidade. No entanto, foi possível identificar quatro fatores, além dos climáticos, que podem estar associados à vulnerabilidade da vegetação de altitude às mudanças climáticas, são eles: a migração altitudinal, os altos índices de endemismo, o degelo antecipado e acelerado e a baixa tolerância a mudanças ambientais em espécies de altitude (Figura1). $\mathrm{O}$ alto índice de endemismo é uma característica dos ambientes de montanhas já bem conhecida e está principalmente associada à heterogeneidade ambiental e a história geológica destes ambientes (Beniston 2006, Martinelli 2007). Apesar de este fator ser frequentemente apontado na literatura como um fator de risco para a vegetação de altitude, poucos trabalhos discutem sobre as causas prováveis da vulnerabilidade de espécies endêmicas a mudanças no clima. Apenas recentemente Hermant et al. (2013) evidenciaram o alto grau de especialização e a forte relação de espécies endêmicas com os ambientes em que ocorrem. Segundo estes autores, isto poderia comprometer a capacidade destas espécies de ocorrer em ambientes diferenciados, além de reduzir a capacidade de resposta a mudanças no clima. Outro fator de risco que merece atenção é o processo de migração altitudinal de espécies de porções mais baixas da montanha para o topo (Colwell et al. 2008), assunto que tem ganhado destaque nos últimos anos. A migração altitudinal é frequentemente apontada como uma das respostas dos organismos às mudanças no clima (Wheng \& Zhou 2006, Engler et al. 2009, Angelo \& Daehler 2013). Segundo Colwell et al. (2008) em decorrência da migração altitudinal, as espécies do topo da montanha estão mais suscetíveis a extinção. Em outras palavras, em um cenário de mudanças no clima onde a migração altitudinal é uma das principais estratégias para manutenção das espécies, aquelas que já estão no topo da montanha não terão para onde ir. É importante destacar que esse e outros processos associados à migração altitudinal provavelmente serão mais intensos em montanhas tropicais. Espera-se que nestas regiões, a migração altitudinal seja mais evidenciada do que a latitudinal, o que não ocorre em montanhas de regiões temperadas (Colwell et al. 2008). Isso acontece porque o gradiente climático altitudinal supera o gradiente latidudinal em regiões tropicais (Colwell et al. 2008). Além disso, é de se esperar que a migração latitudinal seja naturalmente difícil para espécies de topo de montanha como consequência do isolamento geográfico. Alguns trabalhos têm destacado também os impactos que a antecipação e a aceleração do degelo em virtude do aumento da temperatura pode ter sobre essas espécies (Harte et al. 1995), entretanto estes são menos recorrentes (citado em apenas 2 trabalhos consultados nesta revisão). O degelo antecipado acarreta alterações no ciclo hidrológico com 
consequente aumento da estação de crescimento, o que têm afetado a fenologia e produtividade de algumas espécies (Harte et al. 1995). Por último, tem sido observado que algumas espécies de vegetação de montanha podem apresentar baixa tolerância a habitats diferenciados, ou condições ambientais distintas de seus ambientes de ocorrência. A baixa tolerância a mudanças no habitat tem sido observada em plantas de altitude e pode aumentar consideravelmente o risco de extinção (Theurillat \& Guisan, 2001; Thuiller et al. 2004, Kohler et al. 2014). Na revisão de Theurillat \& Guisan (2001) este fator foi destacado como um risco para espécies europeias, e também tem sido mencionado em trabalhos sobre plantas de montanhas tropicais.

\section{OS CAMPOS DE ALTITUDE DO SUDESTE BRASILEIRO EM UM CENÁRIO DE MUDANÇAS CLIMÁTICAS}

No que diz respeito aos efeitos das mudanças climáticas sobre os campos de altitude nenhum estudo foi até agora publicado. Segundo as previsões obtidas através do modelo de circulação geral HadGEM2-ES desenvolvido pela Hadley Centre (The HadGEM2 Development Team 2011) espera-se um aumento na temperatura média anual entre $1,8^{\circ}$ e $2,6^{\circ} \mathrm{C}$ para as regiões do sudeste brasileiro acima de $1800 \mathrm{~m}$ de altitude no ano de 2050. Em relação à precipitação anual, também se prevê um aumento, embora menos dramático, de 25,22 a 109,72 mm para o mesmo ano e faixa altitudinal. Esperam-se também ligeiras alterações na variação anual da temperatura e precipitação para as mesmas regiões (Tabela 1). Além das alterações climáticas graduais e apesar da dificuldade de previsão, espera-se que eventos climáticos extremos, como a ocorrência de secas, tornem-se mais frequentes, sejam de maior magnitude e tenham maior duração (IPCC 2013).

Em geral, nota-se que as principais alterações esperadas são nas médias anuais e não sobre a sazonalidade anual. Com base no trabalho de Barros et al. (2015), que evidencia a sazonalidade climática como um dos fatores determinantes da

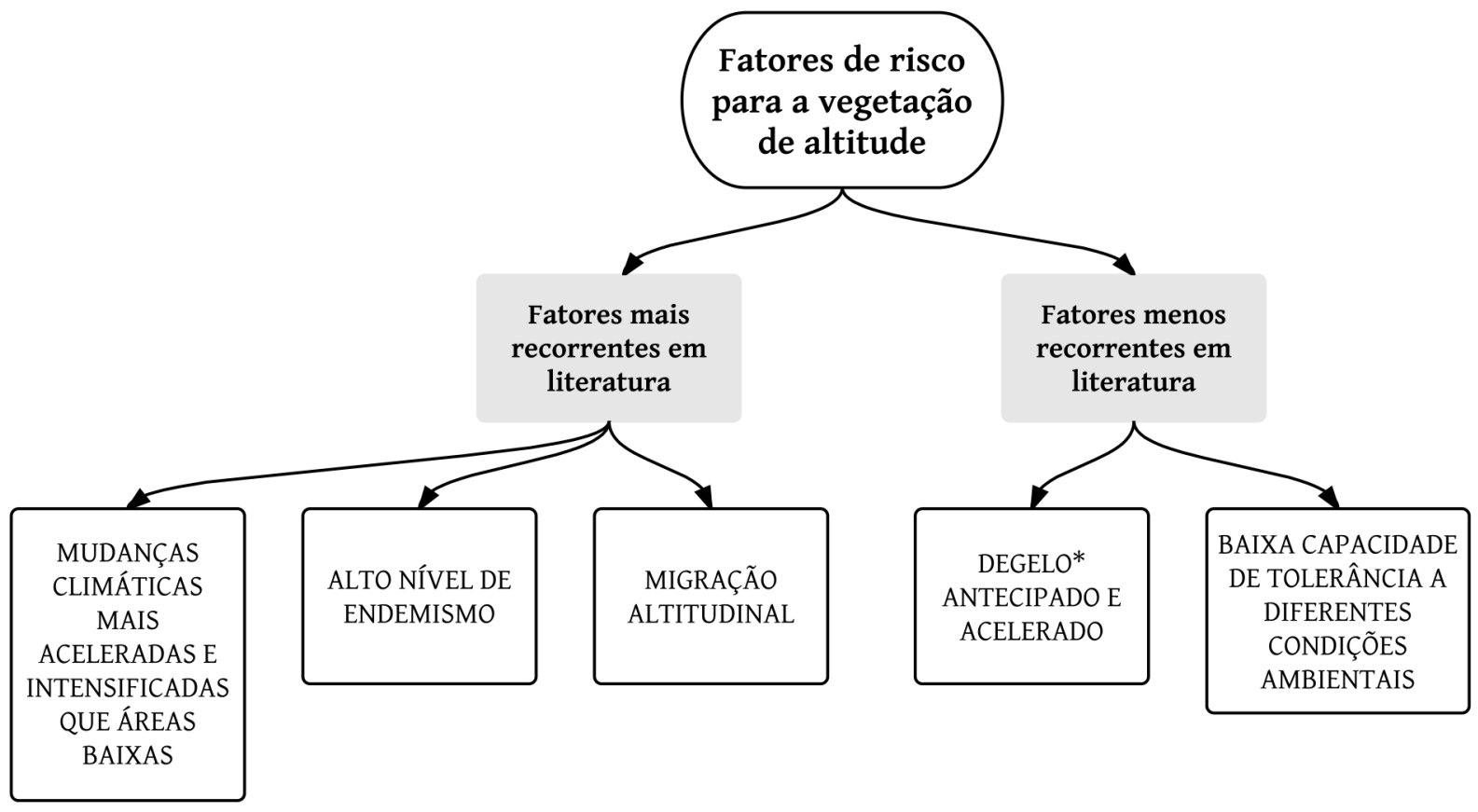

Figura 1. Fatores de risco para a vegetação de altitude classificados de acordo com a frequência de apontamentos na literatura científica consultada nesta revisão. ${ }^{*} \mathrm{O}$ degelo antecipado e acelerado é o único destes fatores que potencialmente não afetaria a vegetação de campos de altitude.

Figure 1. Risk factors for alpine and subalpine vegetation classified according the frequency of literatureconsulted notes. *Anticipated snow break is the only factor that do not affect Brazilian high-altitude vegetation. 
Tabela 1. Previsões de mudanças em 6 variáveis bioclimáticas $(\mathrm{TM}=$ Temperatura Média, $\mathrm{PA}=$ Precipitação anual, TTQ $=$ Temperatura do trimestre mais quente, $\mathrm{TTF}=$ Temperatura do trimestre mais frio, PTS $=$ Precipitação do trimestre mais seco, PTU = precipitação do trimestre mais úmido) para regiões acima de $1800 \mathrm{~m}$ de altitude do Sudeste Brasileiro no ano de 2050 segundo o modelo de circulação Geral HadGEM2ES. São apresentados na tabela os cenários mais otimista (RCP 2.6) e pessimista (RCP 8.5) de emissão de gases segundo o IPCC (2014).

Table 1. Forecast of changes in 6 bioclimatic variables $(T M=$ Annual Mean Temperature, $P A=$ Annual Precipitation, TTQ $=$ Mean Temperature of Warmest Quarter, TTF $=$ Mean Temperature of Coldest Quarter, PTS = Precipitation of Driest Quarter, PTU = Precipitation of Wettest Quarter) for regions above $1800 \mathrm{~m}$ a.s.l. at Brazilian Southeastern in 2050 according to general circulation model HadGEM2-ES. We presented the optimistic (RCP 2.6) and pessimist (RCP 8.5) scenarios of gases emissions according IPCC (2014).

\begin{tabular}{ccccccc}
\hline Cenário & TM $\left(\mathbf{C}^{\mathbf{o}}\right)$ & PA $(\mathbf{m m})$ & TTQ $\left(\mathbf{C}^{\mathbf{0}}\right)$ & TTF $\left(\mathbf{C}^{\mathbf{0}}\right)$ & PTS $(\mathbf{m m})$ & PTU $(\mathbf{m m})$ \\
\hline Atual & 12,68 & 2015,33 & 14,88 & 9,97 & 129,36 & 972,31 \\
RCP 2.6 & 14,48 & 2040,55 & 16,7 & 11,55 & 135,86 & 926,21 \\
RCP 8.5 & 15,28 & 2125,04 & 17,5 & 12,33 & 129,28 & 990,37 \\
\hline
\end{tabular}

biodiversidade vegetal em campos subtropicais do Brasil, acreditamos que futuras alterações nestas variáveis apresentariam potencialmente uma influência maior sobre a vegetação de campos de altitude. Além disso, segundo as análises realizadas neste trabalho e ao contrário do relatado para ambientes de montanha em geral, haverá um incremento maior na temperatura dos meses mais quentes do que naqueles mais frios. Entretanto, é importante ressaltar que o aumento da temperatura do inverno poderá ser alto, na ordem de $1,58^{\circ}$ a $2,36^{\circ} \mathrm{C}$. Do ponto de vista fisiológico, um aumento superior a $2^{\circ} \mathrm{C}$ na temperatura pode ser suficiente para afetar negativamente espécies alpinas (Theurillat \& Guisan 2001). No entanto, não foram encontrados estudos específicos para a vegetação de campos de altitude da região sudeste do Brasil. Além disso, a combinação de altas temperaturas com o aumento da precipitação merece atenção, uma vez que estas seriam as condições ideais para a chegada de novas espécies (como invasoras) e também para o favorecimento do aumento na distribuição altitudinal de espécies arbóreas (Theurillat \& Guisan 2001). Esta chegada de novas espécies poderia promover um impacto negativo para as espécies nativas, através da intensificação da competição (Gottfried et al. 1999). Isso está de acordo com o observado para períodos quentes e úmidos do quaternário, quando havia redução da vegetação de campos de altitude e progressão da floresta atlântica (Behling et al. 2007, Behling \& Safford 2010). A expansão da Floresta Atlântica também é esperada para o futuro de acordo com as previsões atuais de resposta do Bioma às mudanças no clima (Nobre et al. 2007). De acordo com Behling \& Safford (2010), a floresta atlântica estaria atualmente em um período natural de expansão, uma vez que estamos em um período interglacial. Entretanto, em um cenário de mudanças climáticas, esta expansão poderia ocorrer de forma acelerada, comprometendo as formações de campos de altitude (Behling \& Safford 2010). Já é sabido que os campos são favorecidos em período secos e frios, onde apresentam sua extensão máxima, quando deixam de apresentar distribuição disjunta e conectam-se uns com os outros (Behling et al. 2007, Behling \& Safford 2010).

É importante destacar, no entanto, que os campos de altitude se distinguem dos Alpes e de outras montanhas tropicais como os Andes em aspectos determinantes para a resposta da vegetação a mudanças no clima. Estas particularidades estão associadas principalmente a uma sazonalidade fortemente marcada, clima mais frio em uma mesma faixa altitudinal e diferenças florísticas (Safford 1999b, Safford 2007). A ausência de uma "treeline" bem definida também é uma delas. Não se sabe até que ponto a temperatura seria um fator 
limitante para a ocorrência de espécies arbóreas nestes ambientes, prejudicando seu estabelecimento em altitudes mais elevadas. Os baixos valores nutricionais encontrados nos solos de campos de altitude, além de características estruturais e a profundidade do solo (Benites et al. 2003, 2007) podem ter um papel importante na transição da vegetação florestal e da vegetação campestre. Portanto, deste ponto de vista, o impacto da chegada de espécies florestais seria minimizado. Outra diferença marcante é a ausência de uma cobertura significativa de neve ou gelo, que em outras regiões tem sido apontada como tendo uma grande influência na alteração do ciclo hidrológico e nas respostas das espécies. Entretanto, a proximidade dos campos com o oceano pode sim proporcionar um efeito maior das alterações climáticas nestas formações, devido à grande influência exercida da continentalidade sobre o clima em ambientes de montanha (Beniston 2006, Behling 2010). As previsões de alterações climáticas podem ter seu efeito amplificado quando combinadas também a particularidades não climáticas do ambiente e da vegetação de campos de altitude. Especificamente no caso dos campos de altitude da região sudeste do Brasil, que estão frequentemente sujeitos a ocorrência de incêndios antrópicos (Safford 2001, Aximoff \& Rodrigues 2011), bem como sujeitos aos efeitos da expansão acelerada da atividade humana em seus arredores (Ribeiro \& Freitas 2010). Os altos índices de endemismo observados nestes ambientes (Martinelli 1996, Safford 1999a) também podem contribuir para um impacto mais negativo das mudanças climáticas.

\section{FATORES ENDÓGENOS DE VULNERABILIDADE DA VEGETAÇÃODE CAMPOS DE ALTITUDE ÀS MUDANÇAS CLIMÁTICAS}

Nesta revisão não foi encontrado nenhum trabalho realizado em campos de altitude dentro da temática mudanças climáticas. Apesar disso, verifica-se que são recorrentes os apontamentos de que os campos de altitude são altamente vulneráveis às mudanças climáticas em literatura especializada sobre este tipo de formação (i.e., Safford 2001, Martinelli 2007, Behling \& Safford 2010, Ribeiro \& Freitas 2010, entre outros). Aqui, propomos três possíveis fatores e sintetizamos as características da vegetação que contribuem para esta suscetibilidade. O primeiro está associado à distribuição geográfica restrita e disjunta dos campos de altitude; o segundo está associado às características de alta flamabilidade da vegetação e o terceiro e último está relacionado aos altos índices de endemismo comuns a estas formações.

Os campos de altitude encontram-se isolados nos picos das montanhas (Safford 1999a, Longhi-Wagner et al. 2012), apresentando assim uma extensão geográfica restrita e disjunta, correspondendo a menos de $350 \mathrm{Km}^{2}$ (Safford 1999a, Safford 2007). Além da pequena extensão de ocorrência, diferentemente de outras vegetações de montanhas, os campos de altitude encontram-se em restritas faixas altitudinais. No Parque Nacional da Bocaina, por exemplo, estas formações se iniciam por volta dos $1600 \mathrm{~m}$ e chegam até ca. de $2000 \mathrm{~m}$ de altitude. Estes fatores, isto é, sua distribuição disjunta, curta extensão geográfica e ocorrência em uma estreita faixa altitudinal, aumentam a vulnerabilidade da vegetação e das espécies em geral que ocorrem nestes ambientes. Segundo Colwell et al. (2008), as espécies ocorrentes nos topos das montanhas são mais suscetíveis a extinções, processo denominado de "mountaintop extinctions" ou "extinções de topo de montanha". Desta perspectiva, as espécies de campos de altitude que atualmente já ocorrem nas regiões de topo de montanha não teriam para onde migrar e se extinguiriam localmente. Nestas circunstâncias as espécies raras, que correspondem a maior parte das espécies nestes ambientes (Longhi-Wagner et al. 2012), apresentariam maior risco de extinção, uma vez que espécies abundantes e de ampla distribuição geográfica se concentrariam em refúgios (Gottfried 1999, Theurillat \& Guisan 2001). Além disso, a redução da distribuição geográfica das espécies esperada neste cenário (Engler et al. 2009, Mekasha et al. 2013) culminaria também na redução da extensão dos campos de altitude, tornando-os ainda mais ameaçados. Atualmente, já se sabe, por exemplo, que os biomas Andinos que podem apresentar maior perda de área em decorrência das mudanças climáticas são aqueles associados aos topos das montanhas, como o caso dos Páramos, vegetação similar aos campos de altitude (Tovar et al. 2013). 
Características estruturais que conferem alta flamabilidade em espécies ocorrentes nos campos de altitude, como as observadas nas espécies dominantes Chusquea spp. e Cortaderia spp., podem ser compreendidas como um fator de risco para estas formações em um cenário de mudanças climáticas. Neste panorama, a intensificação de ocorrência, duração e magnitude de eventos de seca combinados com o aumento da temperatura podem potencializar a ocorrência de incêndios e/ou tornálos mais devastadores. Apesar dos incêndios serem um importante fator para a manutenção e geração de paisagens alpinas (Safford 2001), o aumento da intensidade e frequência de sua ocorrência podem acarretar mudanças significativas na composição da vegetação de campos de altitude (Safford 2001). Alterações dramáticas na composição da comunidade associadas à ocorrência de incêndios já foram registradas para mudanças no clima do passado nos campos do sul do país (Behling \& Pillar 2007). Além disso, a intensificação de incêndios associada ao aumento da temperatura pode promover a migração altitudinal e aumento de espécies $\mathrm{C}_{4}$ e invasoras (Angelo \& Daehler 2013). No caso dos campos de altitude, espécies como Baccharis reticularia mostram grande sensibilidade à ocorrência de incêndios, bem como baixa capacidade de recuperação, como o caso de Vanillosmopsis erythropappa e Myrsine gardneriana (Safford 2001). Segundo Safford (2001), a intensificação de incêndios nos campos de altitude pode levar a perda de espécies sensíveis, como o caso de $B$. reticularia. Por outro lado, a intensificação dos incêndios pode ser um fator importante para impedir o avanço de espécies arbóreas sobre áreas de campos de altitude, tendo assim um papel importante na manutenção do tipo vegetacional campestre, como já evidenciado por Behling \& Pillar (2007), para os campos do Sul. Atualmente, o aumento da ocorrência de incêndios em campos de altitude do estado do Rio de Janeiro é notório, estando sua maior parte concentrados na estação seca (Aximoff \& Rodrigues 2011).

Assim como as demais formações vegetais associadas às montanhas, os campos de altitude são caracterizados pelos altos níveis de endemismo (Safford 1999a, Aximoff 2011). Como já apontado aqui, este é um dos principais fatores relacionados à vulnerabilidade da vegetação de altitude frente às mudanças climáticas. $\mathrm{O}$ fato de algumas espécies típicas destas formações apresentarem sua distribuição geográfica restrita aos campos de altitudes é preocupante, como anteriormente ressaltado. O número de endemismos restritos, isto é, espécies que estão confinadas a uma única área, também é significativo chegando a mais de $10 \%$ do total de espécies vegetais em algumas localidades de campos de altitude (Martinelli 1996). Este é o caso de Galtheria sleumeriana (Ericaceae) e Hindsia glabra (Rubiaceae) que ocorrem isoladas respectivamente na Serra da Bocaina e no Planalto do Itatiaia, onde apresentam população com reduzido número de indivíduos (Kinoshita-Gouvêa 1981, Freitas et al. 2006, Aximoff 2011) e por isso podem ser afetadas negativamente pelas alterações no clima. A suscetibilidade de espécies endêmicas provavelmente está associada à forte relação que estas espécies apresentam com o ambiente em que evoluíram e que ocorrem (Hermant et al. 2013). Esta alta especialização está relacionada a um alto grau de correlação entre as características funcionais (i.e., alta integração fenotípica) dessas espécies e das espécies por sua vez com o ambiente, comprometendo assim a capacidade de resposta às mudanças ambientais (Hermant et al. 2013). Já existem evidências de que a alta integração fenotípica está relacionada à baixa plasticidade fenotípica (Gianolli \& Palácio-López, 2009), que tem sido associada à vulnerabilidade de plantas a mudanças no clima (Liancourt et al. 2015). Logo, em um cenário de mudanças climáticas, onde se espera que as condições ideais para a ocorrência das espécies sejam modificadas, essas espécies devem ser afetadas negativamente (Hermant et al. 2013). Como nos campos de altitude a ocorrência de espécies endêmicas é significativa, correspondendo a até 33,6\% (endêmicas de campos de altitude e endêmicas restritas) do total de espécies ocorrentes nestas formações (Martinelli 1996), a comunidade vegetal como um todo pode sofrer grande impacto com as mudanças climáticas previstas para o futuro.

\section{CONSIDERAÇÕES FINAIS}

Este estudo foi capaz de sintetizar alguns dos possíveis fatores associados à vulnerabilidade da vegetação de campos de altitude às mudanças 
climáticas. Sendo estes principalmente associados as suas particularidades geográficas, como a distribuição restrita e disjunta, e particularidades biológicas, como a alta flamabilidade da vegetação e os altos índices de endemismo. Entretanto, estudos que busquem avaliar o comportamento de espécies vegetais de altitude em um cenário de alterações climáticas ainda são incipientes, o que compromete a compreensão e a capacidade de previsão de resposta das mesmas. Ainda é necessário avançar neste sentido, por isto aqui sugerimos algumas diretrizes para melhoria do entendimento de como a vegetação de campos de altitude será afetada pelas mudanças futuras no clima (Figura 2). Estudos manipulativos e de modelagem de distribuição de espécies são essenciais para se compreender os tipos de resposta de plantas no cenário futuro, sejam elas associadas às capacidades de tolerância a tais alterações ou a respeito de mudanças em suas distribuições geográficas. É importante destacar também que estudos de ecologia são inexistentes para a maioria das espécies de plantas de campos de altitude. Isto prejudica a capacidade de diferenciação nas respostas entre espécies ou grupos funcionais às mudanças previstas. Deste ponto de vista, estudos com abordagem funcional são bastante úteis. Outro fator que precisa ser levado em consideração são as

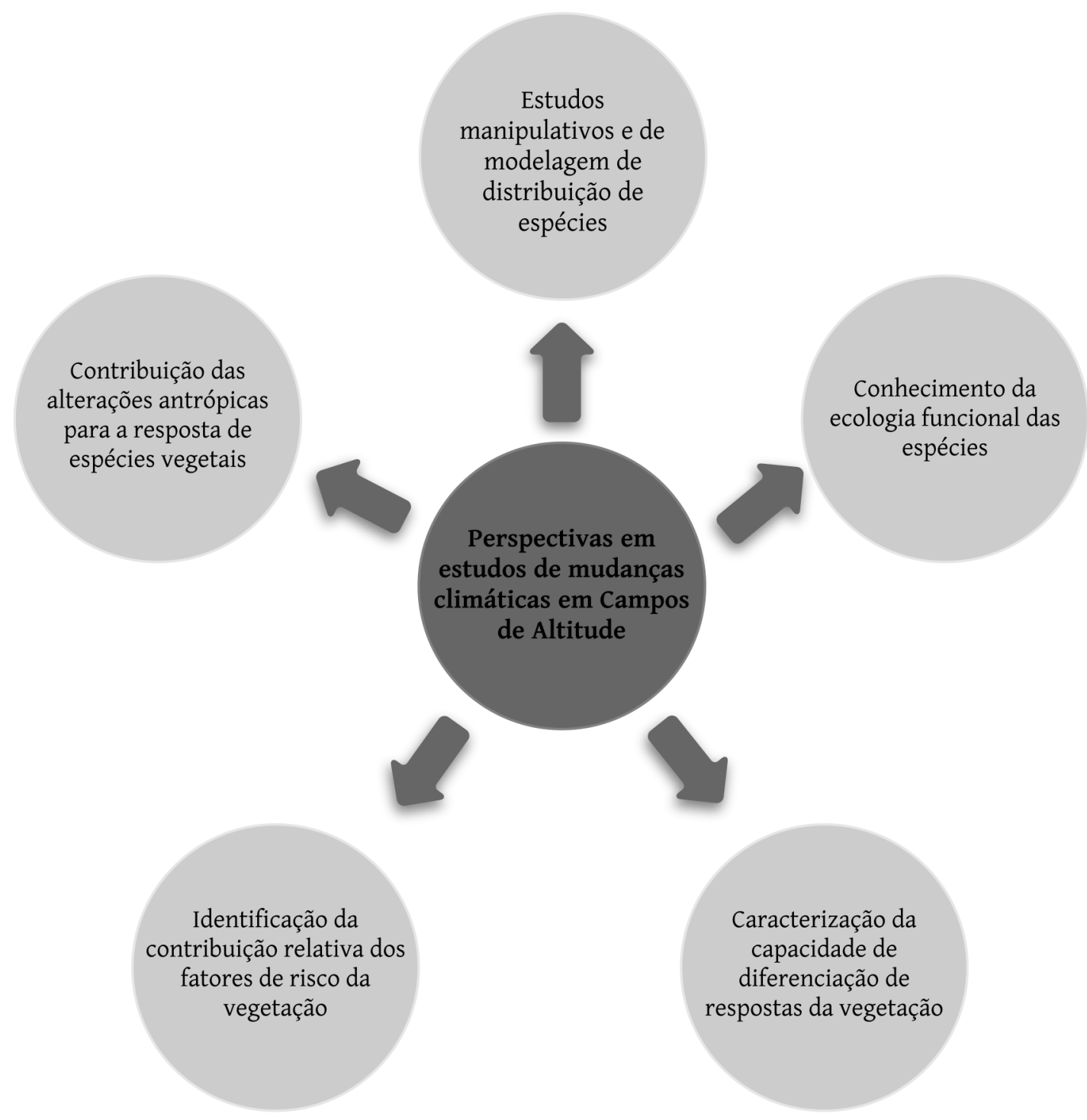

Figura 2. Diretrizes para estudos futuros acerca da influência das mudanças climáticas sobre a vegetação de campos de altitude.

Figure 2. Guidelines for future studies about climate change at Brazilian High-Altitude Grasslands. 
pressões antrópicas as quais estes ambientes estão expostos, como a fragmentação, poluição química e a expansão da agropecuária. Em conjunto com as alterações climáticas esperadas para o futuro, estes fatores podem ser ainda mais agressivos para as espécies de campos de altitude. Além disso, espera-se um futuro avanço altitudinal da zona de agricultura próximo a regiões de campos de altitude com os aumentos da temperatura (Ribeiro \& Freitas 2010). Estas previsões tornam essenciais à realização de estudos aprofundados sobre a ecologia das espécies de campos de altitude. Por fim, é necessário mover esforços para uma identificação mais precisa da contribuição relativa dos diferentes fatores de risco da vegetação de altitude, e especialmente da vegetação de campos de altitude, associados à sua provável vulnerabilidade às mudanças climáticas.

\section{AGRADECIMENTOS}

Nós agradecemos a Alan G. Braz pelo auxílio na compilação de dados do WorldClim para as previsões de mudanças climáticas em Campos de Altitude. A primeira autora também agradece a bolsa PDS concedida pela CAPES para realização de seu Mestrado.

\section{REFERÊNCIAS}

Angelo, C. L. \& Daehler, C.C. 2013. Upward expansion of fire-adapted grasses along a warming tropical elevation gradient. Ecography, 36(5), 551-559. DOI: 10.1111/j.1600-0587.2012.07754.x

Aximoff, I. 2011. O que Perdemos com a Passagem do Fogo pelos Campos de Altitude do Estado do Rio de Janeiro? Biodiversidade Brasileira - Número Temático sobre Ecologia e Manejo de Fogo em Áreas Protegidas. ICMBio, 2(1), 180 - 200.

Aximoff, I. \& Rodrigues, R. de C. 2011. Histórico dos incêndios florestais no parque nacional do Itatiaia. Ciência Florestal, 21(1), 83-92. DOI: 10.5902/198050982750

Baron, J. S., Hartman, M. D., Band, L. E., Lammers, R. B. 2000. Sensitivity of a High-Elevation Rocky Mountain Watershed to Altered Climate and $\mathrm{CO}_{2}$. Water Resources Research, 36(1), 89-99. DOI: 10.1029/1999WR900263

Barros, M. J. F., Silva-Arias, G. A., Fregonezi, J. N., Turchetto-Zolet, A. C., Iganci, J. R. V., Diniz-Filho, J. A. F., Freitas, L. B. 2015. Environmental drivers of diversity in Subtropical Highland Grasslands. Perspectives in Plant Ecology, Evolution and Systematics, 17(5), 360-368. DOI: 10.1016/j.ppees.2015.08.001

Behling, H. \& Safford, H. D. 2010. Late-Glacial and Holocene Vegetation, Climate and Fire Dynamics in the Serra Dos Órgãos, Rio de Janeiro State, Southeastern Brazil. Global
Change Biology,16(6), DOI: 1661-1671.10.1111/j.13652486.2009.02029.x

Behling, H., Dupont, L., Safford, H. D., Wefer, G. 2007. Late Quaternary Vegetation and Climate Dynamics in the Serra Da Bocaina, Southeastern Brazil. Quaternary International, 161(1), 22-31. DOI: 10.1016/j.quaint.2006.10.021

Behling, H. \& Pillar, V. D. 2007. Late Quaternary vegetation, biodiversity and firedynamics on the southern Brazilian highland and their implication for conservation andmanagement of modern Araucaria forest and grassland ecosystems. Philosophical Transactions of the Royal Society B, 362(1478), 243-251. DOI: 10.1098/ rstb.2006.1984

Bellard, C., Bertelsmeier, C., Leadley, P., Thuiller, W., Courchamp, F. 2012. Impacts of climate change on the future of biodiversity. Ecology Letters, 15(4), 365-377. DOI: $10.1111 / \mathrm{j} .1461-0248.2011 .01736 . x$

Beniston, M.; Diaz, H. F., Bradley, R. S. 1997. Climatic Change at High Elevation Sites: An Overview. Climatic Change, 36(3), 233-251. DOI: 10.1023/A:1005380714349

Beniston, M. 2006. Mountain Weather and Climate: A General Overview and a Focus on Climatic Change in the Alps. Hydrobiologia, 562(1), 3-16. DOI: 10.1007/ s10750-005-1802-0

Beniston, M. \& Stoffel, M. 2013. Assessing the Impacts of Climatic Change on Mountain Water Resources. The Science of the Total Environment, 493(1),1129-1137. DOI: 10.1016/j.scitotenv.2013.11.122

Benites, V. M., Caiafa, A. N. ,Mendonça, E. S., Schefer, C. E., Ker, J. C. 2003. Solos e vegetação nos complexos rupestres de altitude da Mantiqueira e do Espinhaço. Floresta e Ambiente, 10(1), 76-86.

Benites, V. M., Schaefer, C. E. G. R., Simas, F. N. B., Santos, H. G. 2007. Soils associated with rock outcrops in the Brazilian mountain ranges Mantiqueira and Espinhaço. Revista Brasileira de Botânica, 30(4), 569-577. DOI: 10.1590/S0100-84042007000400003

Colwell, R. K., Brehm, G., Cardelús, C. L., Gilman, A. C., Longino, J. T. 2008. Global warming, elevational range shifts, and lowland biotic attrition in the wet tropics. Science, 322(5899), 258-61. DOI: 10.1126/ science. 1162547

CONABIO. 2008. Deliberação n ${ }^{\circ} 57$, de 28 de outubro de 2008. Dispõe sobre a criação da Câmara Técnica Temporária sobre Ecossistemas de Montanha. http://www.mma.gov. br/estruturas/conabio/_arquivos/deliberacao_57_15.pdf. Acesso em 06 de outubro de 2015.

Engler, R., Randin, C. F., Vittoz, P., Czáka, T., Beniston, M., Zimmermann, N. E., Guisan, A. 2009. Predicting Future Distributions of Mountain Plants under Climate Change: Does Dispersal Capacity Matter? Ecography, 32(1), 3445. DOI: 10.1111/j.1600-0587.2009.05789.x

Engler, R., Randin, C. F., Thuiller, W., Dullinger, S., Zimmermann, N. E., Araújo, M. B., Pearman, P. B., Lay, G. L., Piedallu, C., Albert, C. H., Choler, P., Coldea, G., de Lamo, X., Dirnböck, T., Gégout, J. C., Gómez-Garcia, D., Grytnes, J. A., Heegaard, E., Hoistad, F., Nogués- 
Bravo, D., Normand, S., Puscas, M., Sebastià, M. T., Stanisci, A., Theurillat, J. P., Trivedi, M. R., Vittoz, P., Guisan, A. 2011. 21st century climate change threatens mountain flora unequally across Europe. Global Change Biology, 17(1), 2330-2341. DOI: 10.1111/j.13652486.2010.02393.x

Gottfried, M., Pauli, H., Reiter, K., Grabherr, G. 1999. A Fine-Scaled Predictive Model for Changes in Species Distribution Patterns of High Mountain Plants Induced by Climate Warming. Diversity and Distributions, 5(6), 241-51. DOI: 10.1046/j.1472-4642.1999.00058.x

Freitas, L., Galetto, L. \& Sazima, M. 2006. Pollination by hummingbirds and bees in eight syntopic species and a putative hybrid of Ericaceae in southeastern Brazil. Plant Systematics and Evolution, 258(1), 49-61. DOI: 10.1007/ s00606-005-0392-7

Gianolli, E. \& Palacio-López, K. 2009. Phenotypic integration may constrain phenotypic plasticity in plants. Oikos, 118(12), 1924-1928. DOI: 10.1111/j.16000706.2009.17884.x

Hannah, Lee. 2011. Climate Change Biology. Academic Press, London: 402p.

Harte, J. \& Shaw, R. 1995. Shifting Dominance within a Montane Vegetation Community : Results of a ClimateWarming Experiment. Science, 267(5199), 876-80. DOI: $10.1126 /$ science. 267.5199 .876

The HadGEM2 Development Team. 2011. The HadGEM2 family of Met Office Unified Model climate configurations. Geoscientific model development, 4(1), 723-757. DOI: 10.5194/gmd-4-723-2011

Hermant, M., Prinzing, A., Vernon, P., Convey, P., Hennion, F. 2013. Endemic species have highly integrated phenotypes, environmental distributions and phenotypeenvironment relationships. Journal of Biogeography, 40(8), 1583-1594. DOI: 10.1111/jbi.12095

Hijmans, R. J. 2015. raster: Geographic data analysis and modeling (R package). (http://ouch.r-forge.r-project.org)

IPCC (Intergovernmental Panel on Climate Changes) 2013. Climate change 2013: The physical science basis. Contribution of Working Group I to the Fifth Assessment Report of the Intergovernmental Panel on Climate Change [Stocker, T.F., D. Qin, G.-K. Plattner, M. Tignor, S.K. Allen, J. Boschung, A. Nauels, Y. Xia, V. Bex and P.M. Midgley (eds.)]. Cambridge University Press, Cambridge, Reino Unido e Nova York: 1535p.

Kinoshita-gouvêa, L. S. 1981. Novas espécies de Ericaceae para o Brasil: Gaultheriaslemeuriana, Leucothoechapadensis e Gaylussaciasetosa. Revista Brasileira de Botânica, 4(2), 125-130.

Kohler, T., Giger, M., Hurni, H., Ott, C., Wiesmann, U., von Dachand, S. W., Maselli, D. 2010. Mountains and Climate Change: A Global Concern. Mountain Research and Development, 30(1), 53-55.

Kohler, T., Wehrli, A. \& Jurek, M. 2014. Mountains and climate change: A global concern. Sustainable Mountain Development Series. Bern, Switzerland, Centre for Development and Environment (CDE), Swiss Agency for
Development and Cooperation (SDC) and Geographica Bernensia: $136 \mathrm{p}$.

Kreyling, J., Wana, D. \& Beierkuhnlein, C. 2010. Potential Consequences of Climate Warming for Tropical Plant Species in High Mountains of Southern Ethiopia. Diversity and Distributions, 16(4), 59-605. DOI: 10.1111/j.1472-4642.2010.00675.x

Liancourt, P., Boldgiv, B., Song, D. S., Spence, L. A., Helliker, B. R., Petraitis, P. S., Casper, B. B. 2015. Leaftrait plasticity and species vulnerability to climate change in a Mongolian steppe. Global Change Biology, 21(9), 3489-3498. DOI: $10.1111 / \mathrm{gcb} .12934$

Longhi-wagner, H. M., Welker, C. A. D. \& Waechter, J. L. 2012. Floristic Affinities in Montane Grasslands in Eastern Brazil. Systematics and Biodiversity, 10(4), 537-550. DOI: 10.1080/14772000.2012.753487

Malhi, Y., Roberts, J. T., Betts, R. A., Killeen, T. J., Li, W., Nobre, C. A. 2008. Climate Change, Deforestation and the Fate of the Amazon. Science, 319(5860), 169-172. DOI: $10.1126 /$ science. 1146961

Marengo, J. A.; 2006. Mudanças climáticas globais e seus efeitos sobre a biodiversidade: Caracterização do clima atual e definição das alterações climáticas para o território brasileiro ao longo do século XXI. Instituto Brasileiro do Meio Ambiente e dos Recursos Naturais Renováveis, Brasília: 212p.

Martinelli, G. 1996. Campos de Altitude: High mountain grasslands. Editora Index, Rio de Janeiro, 160p.

Martinelli, G. 2007. Mountain Biodiversity in Brazil. Revista Brasileira de Botânica, 30(4), 587-597. DOI: 10.1590/ S0100-84042007000400005

Mekasha, A., Nigatu, L., Tesfayeand, K., Duncan, A. J. 2013. Modeling the response of tropical highland herbaceous grassland species to climate change: The case of the Arsi Mountains of Ethiopia. BiologicalConservation, 168(1), 169-175. DOI: 10.1016/j.biocon.2013.09.020

Nobre, C. A., Salazar, L. F., Oyama, M., Cardoso, M., Sampaio, G., Lapola, D. (2007) Mudanças Climáticas Globais e Efeitos sobre a Biodiversidade. Sub projeto: Caracterização do clima atual e definição das alterações climáticas para o território brasileiro ao longo do Século XXI Relatório No. 6 Mudanças Climáticas e possíveis alterações nos Biomas da América do Sul. Ministério do Meio Ambiente, São Paulo, Brasil.

PBMC. 2014. Base científica das mudanças climáticas. Contribuição do Grupo de Trabalho 1 do Painel Brasileiro de Mudanças Climáticas ao Primeiro Relatório da Avaliação Nacional sobre Mudanças Climáticas [Ambrizzi, T., Araujo, M. (eds.)]. COPPE. Universidade Federal do Rio de Janeiro, Rio de Janeiro, RJ, Brasil: 464 pp.

Pounds, J. A., Fogden, M. P. L. \& Campbell, J. H. 1999. Biological Response to Climate Change on a Tropical Mountain. Nature, 398, 611-615. DOI: 10.1038/19297

Rangwala, I. \& Miller, J. R. 2012. Climate Change in Mountains: A Review of Elevation-Dependent Warming and Its Possible Causes. Climatic Change, 114(3), 527547. DOI: $10.1007 / \mathrm{s} 10584-012-0419-3$ 
Ribeiro, K. T. \& Freitas, L. 2010. Impactos Potenciais Das Alterações No Código Florestal Sobre a Vegetação de Campos Rupestres e Campos de Altitude. Biota Neotropica, 10(4), 239-246. DOI: 10.1590/S167606032010000400029

Safford, H. D. 1999a. Brazilian Páramos I. An Introduction to the Brazilian Physical Environment and Vegetation of the Campos de Altitude. Journal of Biogeography, 26(4), 693-712. DOI: 10.1046/j.1365-2699.1999.00313.x

Safford, H. D. 1999b. Brazilian Páramos II. Macro- and Mesoclimate of Campos de Altitude and Affinities with High Mountain Climates of the Tropical Andes and Costa Rica. Journal of Biogeography, 26(4), 713-737. DOI: 10.1046/j.1365-2699.1999.00312.x

Safford, H. D. 2001. Brazilian Páramos. III. Patterns and rates of post fire regeneration in the campos de altitude. Biotropica, 33(2), 282-302. DOI: 10.1646/0006-3606(2001)033[0282:BPRIPA]2.0.CO;2

Safford, H. D. 2007. Brazilian Páramos IV. Phytogeography of the Campos de Altitude. Journal of Biogeography, 34(10), 1701-1722. DOI: 10.1111/j.1365-2699.2007. 01732.x

Sanz-Elorza, M., Dana, E. D., González, A., Sobrino, E. 2003. Changes in the High-Mountain Vegetation of the Central Iberian Peninsula as a Probable Sign of Global Warming. Annals of Botany, 92(4), 273-280. DOI: 10.1093/aob/mcg130

Shaw, M. R., Loik, M. E., Harte, J. 2000. Gas Exchange and Water Relations of Two Rocky Mountain Shrub Species Exposed to a Climate Change Manipulation. Plant Ecology, 146(2), 197-206. DOI:10.1023/A:1009863201751
Theurillat, J. \& Guisan, A. 2001. Potential impact of climate change on vegetation in the European alps: a review. Climatic Change, 50(1), 77-109. DOI: 10.1023/A:1010632015572

Thuiller, W., Lavorel, S., Araújo, M. B., Sykes, M. T., Prentice, C. 2005. Climate change threats to plant diversity in Europe. PNAS, 102(22), 8245-8250.

Tovar, C., Arnillas, C. A., Cuesta, F., Buytaert, W. 2013. Diverging Responses of Tropical Andean Biomes under Future Climate Conditions. PlosOne, 8(5), 1-12. DOI: 10.1371/journal.pone.0063634

Vale, M. M., Alves, M. A. S. \& Lorini, M. L. 2009. Mudanças Climáticas: Desafios e oportunidades para a conservação da biodiversidade brasileira. OecologiaAustralis, 13(3), 518-535.

Van Vuuren, D. P., Edmonds, J., Kainuma, M., Riahi, K., Thomson, A., Hibbard, K., Hurtt, G. C., Kram, T., Krey, V., Lamarque, J. F., Masui, T., Meinshausen, M., Nakicenovic, N., Smith, S. J. ,Rose, S. K. 2011. The representative concentration pathways: an overview. Climatic Change, 109(5), 5-31. DOI: $10.1007 / \mathrm{s} 10584-$ 011-0148-z

Vuille, M. \& Bradley, R. 2000. Mean annual temperature trends and their vertical structure in the tropical Andes. Geophysical Research Letters, 27(23), 3885-3888. DOI: 10.1029/2000GL011871

Weng, E. \& Zhou, G. 2006. Modeling distribution changes of vegetation in China under future climate change. Environmental Modeling and Assessment, 11(1), 45-58. DOI: $10.1007 / \mathrm{s} 10666-005-9019-1$ 